\title{
Increased Monocyte/Lymphocyte Ratio as Risk Marker for Cardiovascular Events and Infectious Disease Hospitalization in Dialysis Patients
}

\author{
Reiko Muto $^{a}$ Sawako Kato ${ }^{a}$ Bengt Lindholm ${ }^{b}$ Abdul Rashid Qureshi ${ }^{b}$ \\ Takuji Ishimoto $^{a}$ Tomoki Kosugi $^{a}$ Shoichi Maruyama ${ }^{a}$ \\ aDepartment of Nephrology, Nagoya University Graduate School of Medicine, Nagoya, Japan; baxter Novum \& \\ Renal Medicine Karolinska Institutet, Stockholm, Sweden
}

\section{Keywords}

Monocyte/lymphocyte ratio - Cardiovascular disease . Infectious disease $\cdot$ Dialysis

\begin{abstract}
Introduction: In dialysis patients, cardiovascular disease (CVD) and infectious disease contribute to poor clinical outcomes. We investigated if a higher monocyte/lymphocyte ratio (MLR) is associated with an increased risk of CVD events and infectious disease hospitalizations in incident dialysis patients. Methods: In an ongoing observational prospective cohort study, 132 Japanese dialysis patients (age 58.7 \pm 11.7 years; $70 \%$ men) starting dialysis therapy were enrolled and followed up for a median of 48.7 months. Laboratory biomarkers, including white blood cell count and its differential count, were determined at baseline. Event-free time and relative risks (RRs) were calculated using the Kaplan-Meier curves and Cox models, respectively. Results: When divided into 2 groups according to median MLR ( 0.35 [range, 0.27 0.46]), the periods without CVD events were significantly shorter in the high MLR group than in the low MLR group (log-rank test $=5.60, p=0.018$ ). The RR of CVD events, after adjusting for age, sex, and diabetes, was 2.43 (1.22-4.84) in the high MLR group compared to the low MLR group. The periods without infections requiring hospitalization were also shorter (log-rank test $=4.16, p=0.041)$. The RR of infections requiring hospitalization was $1.98(1.02-3.83)$ after the
\end{abstract}

karger@karger.com www.karger.com/bpu

Karger"
(C) 2021 The Author(s)

Published by S. Karger AG, Base

This is an Open Access article licensed under the Creative Common Attribution-NonCommercial-4.0 International License (CC BY-NC) (http://www.karger.com/Services/OpenAccessLicense), applicable to the online version of the article only. Usage and distribution for commercial purposes requires written permission. same adjustments. The number of CVD events was higher in the high MLR group (18.6 events per 100 person-years at risk [pyr]) than the low MLR group (11.1 events per 100 pyr). The duration of infectious disease hospitalization was longer in the high MLR group (6.3 days per pyr) than in the low MLR group (2.8 days per pyr). Conclusion: A higher MLR is associated with increased risks of both CVD events and infectious disease hospitalization in dialysis patients.

(c) 2021 The Author(s)

Published by S. Karger AG, Basel

\section{Introduction}

The survival rate of patients with chronic kidney disease stage 5 undergoing dialysis (CKD-5D) has been improving during recent years, but the risks of mortality and hospitalization still remain much higher than that in the general population $[1,2]$. Cardiovascular disease (CVD) is the leading cause of death among dialysis patients along with a high prevalence of cardiovascular morbidity [3-5]. One of the reasons for the difficulty in reducing the burden of CVD in dialysis patients is the increasing proportion of older and diabetic patients starting renal replacement therapy $[4,6]$.

The high premature mortality in dialysis patients is due not only to CVD but also to non-CVD causes of death, especially infectious complications. Among patients starting dialysis, CVD deaths account for about 
half of the total mortality, while infections account for $20 \%$, malignancies for $10 \%$, and the rest are due to other causes [7]. Of note, rates of infection (which is the second leading cause of death also among younger patients [7]) and infectious conditions requiring hospitalization have not decreased, despite the decline in catheter use in hemodialysis patients [8]. In Japan, although the proportion of hemodialysis patients using catheter is relatively low, mortality due to infectious diseases is markedly higher in dialysis patients than in the general population [9]. Furthermore, CVD and infectious diseases appear to aggravate each other [10], possibly because they share risk factors and potential causal pathways that link CVD with infections and vice versa [11, 12].

We observed in a previous study that a higher neutrophil/lymphocyte ratio (NLR) indicated an increased risk of CVD in incident dialysis patients [13]. However, recent studies suggest that monocyte/lymphocyte ratio (MLR) might be a more sensitive predictor of CVD events than NLR $[14,15]$ because monocytes, which are ancestors of lipid-laden macrophages, may directly contribute to the progression of atherosclerosis $[14,15]$. Moreover, as the monocyte is essentially a professional immune cell belonging to the innate immune system, MLR might be also a predictor of infectious complications in dialysis patients who are susceptible to symptomatic and asymptomatic infections [11]. Therefore, this study aimed to examine the association between MLR and risk of CVD events and hospitalization due to infectious disease in Japanese CKD-5D patients who had recently started dialysis therapy.

\section{Materials and Methods}

\section{Study Design and Patients}

We enrolled 172 stable incident dialysis patients who were 75 years old or younger between June 2007 and December 2014 at Masuko Memorial Hospital and Meiyo Clinic in the Aichi Prefecture, Japan. This study was part of an ongoing prospective cohort study approved by the Ethics Committee of Nagoya University Graduate School of Medicine (No. 2012-0241), details of which have been described in reports based on this cohort [13, 16-18]. Informed consent for participation was obtained from all patients. In the present study, we investigated 132 patients having data of differential leukocyte count out of 172 patients in the whole cohort.

Data Collection, Blood Sampling, and Laboratory Analysis

The procedures were described in our previous reports [13, 16-18]. In brief, baseline demographic and clinical data were collected from the patients' records and assessed during inter- views. Blood samples were collected from each subject within 1 month of the first dialysis session. Blood tests, including complete blood cell count and differential leukocyte count, were performed at the clinical laboratory in each facility using automatic analyzer.

\section{Statistical Analysis}

Continuous variables are presented as mean \pm SD and/or median and interquartile range (25th-75th percentiles). MLR was calculated for all study participants and those with an MLR greater than the median MLR value were grouped in the high MLR group and the rest were in the low MLR group. To evaluate baseline characteristics and laboratory biomarkers, comparisons of continuous parameters between the high MLR and low MLR groups were performed using the Wilcoxon singed-rank test. Nominal variables were tested using the $\chi^{2}$ test. Durations from start of dialysis until the first CVD event and hospitalization by infection were analyzed using the Kaplan-Meier curve, and differences were examined by the log-rank test. The Cox proportional hazard model was used to calculate relative risks (RRs) with 95\% confidence intervals (CIs) of the first CVD event and first hospitalization due to infection after the start of dialysis after adjusting for age, sex, and diabetes mellitus. A $p$ value of $<0.05$ was considered statistically significant. All statistical analyses were performed using statistical software JMP Pro version 15.0.0 (SAS Campus Drive, Cary, NC, USA; 27513).

\section{Results}

\section{Patients Characteristics and Laboratory Biomarkers}

Clinical characteristics and laboratory data at baseline are summarized in Table 1 . In the study cohort of $132 \mathrm{pa}-$ tients (average age $58.7 \pm 11.7$ years; 91 [68.9\%] men), median MLR was 0.35 (range, 0.27-0.46). The baseline prevalence of CVD was significantly higher among patients with higher MLR.

\section{CVD and Infection Outcomes}

We followed the patients until death; transfer to another clinic, renal transplantation, or until end of followup, which ended in December 2014. The median followup period was 48.7 months (range, 1-113 months). During follow-up, patients experienced a total of $84 \mathrm{CVD}$ events and 87 infection-related hospitalizations. The summary of them is shown in Table 2.

\section{The Time Free of CVD Events after Start of Dialysis Therapy}

Although detailed causes of sudden deaths are often unknown, some may be related to hyperkalemia and/or congestive heart failure due to overhydration as well as to other CVD-related causes of sudden death, especially in dialysis patients. Because MLR might contribute to 
Table 1. Clinical characteristics and laboratory biomarkers at baseline in 132 incident dialysis patients

\begin{tabular}{|c|c|c|c|c|}
\hline \multirow[t]{2}{*}{ Variables } & \multirow[t]{2}{*}{ Total } & \multicolumn{2}{|c|}{ MLR (median: 0.35) } & \multirow[t]{2}{*}{$p$ value } \\
\hline & & MLR $>$ median & MLR $<$ median & \\
\hline Number & 132 & 66 & 66 & \\
\hline Male, $n(\%)$ & $91(68.9)$ & $50(75.8)$ & $41(62.1)$ & 0.09 \\
\hline Age, year & $58.7 \pm 11.7$ & $60.5 \pm 9.2$ & $56.8 \pm 13.6$ & 0.21 \\
\hline Body mass index, $\mathrm{kg} / \mathrm{m}^{2}$ & $22.0 \pm 4.0$ & $22.5 \pm 4.6$ & $21.8 \pm 3.4$ & 0.46 \\
\hline Diabetes mellitus, $n$ (\%) & $66(50.0)$ & $33(50.0)$ & $33(50.0)$ & 1.00 \\
\hline \multicolumn{5}{|l|}{ Smoking, $n(\%)$} \\
\hline Current & $25(19.1)$ & $15(23.1)$ & $10(15.2)$ & \multirow{2}{*}{0.13} \\
\hline Ex-smokers & $44(33.6)$ & $25(38.5)$ & $19(28.8)$ & \\
\hline \multicolumn{5}{|l|}{ Medication, $n(\%)$} \\
\hline ACE-I/ARBs & $84(63.6)$ & $43(65.2)$ & $41(62.1)$ & 0.72 \\
\hline Statins & $49(37.4)$ & $21(32.3)$ & $28(42.2)$ & 0.23 \\
\hline Vitamin D & $72(55.0)$ & $41(63.1)$ & $31(47.0)$ & 0.06 \\
\hline ESA & $112(86.2)$ & $53(82.8)$ & $59(89.4)$ & 0.28 \\
\hline \multicolumn{5}{|l|}{ Malnutrition, $n(\%)$} \\
\hline SGA category B (mildly moderately) & $77(60.6)$ & $41(64.1)$ & $36(57.1)$ & \multirow{2}{*}{0.64} \\
\hline SGA category C (severely) & $22(17.3)$ & $11(17.2)$ & $11(17.5)$ & \\
\hline History of CVD & $37(28.0)$ & $26(39.4)$ & $11(26.7)$ & $0.003^{*}$ \\
\hline \multicolumn{5}{|l|}{ Modality of dialysis, $n(\%)$} \\
\hline Hemodialysis & $123(93.2)$ & $60(90.9)$ & $63(95.5)$ & \multirow{2}{*}{0.30} \\
\hline Peritoneal dialysis & $9(6.8)$ & $6(9.1)$ & $3(4.6)$ & \\
\hline CV catheter usage at initiation (HD), $n(\%)$ & $19(15.4)$ & $8(13.3)$ & $11(17.4)$ & 0.53 \\
\hline \multicolumn{5}{|l|}{ Vascular access (HD), $n(\%)$} \\
\hline AV fistula & $111(92.5)$ & $55(93.2)$ & $56(91.8)$ & \multirow{2}{*}{0.77} \\
\hline Graft & $9(7.5)$ & $4(6.8)$ & $5(8.2)$ & \\
\hline Hemoglobin, g/dL & $9.1 \pm 1.4$ & $9.1 \pm 1.4$ & $9.0 \pm 1.4$ & 0.92 \\
\hline WBC $\left(\times 10^{3} / \mathrm{mm}^{3}\right)$ & $6.1 \pm 2.2$ & $6.2 \pm 2.5$ & $5.9 \pm 1.8$ & 0.58 \\
\hline Neutrophils $\left(\times 10^{3} / \mathrm{mm}^{3}\right)$ & $4.2 \pm 1.9$ & $4.4 \pm 2.2$ & $4.0 \pm 1.6$ & 0.22 \\
\hline Lymphocytes $\left(\times 10^{3} / \mathrm{mm}^{3}\right)$ & $1.1 \pm 0.4$ & $1.0 \pm 0.4$ & $1.3 \pm 0.4$ & $<0.0001^{*}$ \\
\hline Monocytes $\left(\times 10^{3} / \mathrm{mm}^{3}\right)$ & $0.4 \pm 0.2$ & $0.5 \pm 0.2$ & $0.3 \pm 0.1$ & $<0.0001^{*}$ \\
\hline Neutrophils (\% of WBC) & $67.5 \pm 8.4$ & $69.3 \pm 8.5$ & $65.8 \pm 8.0$ & $0.012^{*}$ \\
\hline Lymphocytes (\% of WBC) & $19.4 \pm 6.3$ & $16.0 \pm 4.5$ & $22.8 \pm 6.1$ & $<0.0001^{*}$ \\
\hline Monocytes (\% of WBC) & $7.0 \pm 2.4$ & $8.3 \pm 2.4$ & $5.7 \pm 1.4$ & $<0.0001^{*}$ \\
\hline Platelet $\left(\times 10^{3} / \mathrm{mm}^{4}\right)$ & $20.8 \pm 8.2$ & $20.9 \pm 9.9$ & $20.7 \pm 6.1$ & 0.57 \\
\hline Albumin, $\mathrm{g} / \mathrm{dL}$ & $3.4 \pm 0.5$ & $3.4 \pm 0.5$ & $3.4 \pm 0.6$ & 0.74 \\
\hline Total cholesterol, mg/dL & $165.6 \pm 43.4$ & $165.6 \pm 42.5$ & $165.5 \pm 44.9$ & 0.90 \\
\hline HDL cholesterol, mg/dL & $46.8 \pm 14.9$ & $46.4 \pm 15.1$ & $47.1 \pm 14.8$ & 0.74 \\
\hline $\mathrm{UA}, \mathrm{mg} / \mathrm{dL}$ & $7.8 \pm 2.2$ & $8.3 \pm 2.4$ & $7.2 \pm 1.8$ & $0.008^{*}$ \\
\hline Creatinine, mg/dL & $9.5 \pm 3.3$ & $9.8 \pm 3.6$ & $9.1 \pm 2.8$ & 0.44 \\
\hline Intact PTH, pg/mL & $317.2 \pm 238.6$ & $310 \pm 260$ & $324 \pm 217$ & 0.35 \\
\hline Ferritin, ng/mL & $129.5 \pm 158.3$ & $156.9 \pm 101.4$ & $152 \pm 198$ & 0.19 \\
\hline $\mathrm{CRP}, \mathrm{mg} / \mathrm{dL}$ & $0.10(0.04-0.46)$ & $0.11(0.05-0.70)$ & $0.072(0.021-0.30)$ & $0.009^{*}$ \\
\hline
\end{tabular}

ACE-I/ARBs, angiotensin-converting enzyme inhibitor/angiotensin receptor blocker; ESA, erythropoiesisstimulating agents; UA, uric acid; PTH, parathyroid hormone; CRP, C-reactive protein; MLR, monocyte/lymphocyte ratio; CVD, cardiovascular disease.

progression of atherosclerosis, we performed 2 calculations for CVD outcomes, CVD events excluding sudden death - these events may represent causes due to atherogenic disease - and the total number of CVD events. When divided into 2 groups according to me- dian MLR (0.35 [range, 0.27-0.46]), and excluding CVD events caused by sudden deaths, Kaplan-Meier curves of the period without CVD event showed that the period from the start of dialysis therapy to the first such CVD event was significantly shorter in the high 
Table 2. Summary of main underlying causes of CVD events and infection-related hospitalizations

\begin{tabular}{ll}
\hline CVD events $(n=84)$ & $n$ \\
\hline Diseases & 29 \\
\hline Angina & 19 \\
Peripheral arterial disease & 15 \\
Stroke & 9 \\
Sudden death & 7 \\
Myocardial infarction & 3 \\
Transient ischemic attack & 1 \\
Heart failure & \\
\hline Infection-related hospitalization $(n=87)$ & $n$ \\
\hline Diseases & 29 \\
\hline Pneumonia & 11 \\
Infectious gangrene & 11 \\
Enteritis & 9 \\
Sepsis & 8 \\
Vascular access infection & 3 \\
Peritonitis & 3 \\
Urinary tract infection & 3 \\
Infections related to peritoneal dialysis &
\end{tabular}

CVD events and infection-related hospitalization include lethal CVD events and lethal infectious diseases, respectively. CVD, cardiovascular disease.

MLR group than in the low MLR group (log-rank test $=5.59, p=0.018$ ) (Fig. 1a). When evaluating all CVD events including those caused by sudden deaths, Kaplan-Meier curves of the period without any CVD event showed that the period was also significantly shorter in the high MLR group than in the low MLR group (log-rank test $=5.18, p=0.023$ ) (Fig. 1b).

Next, we calculated the RRs of the first CVD event in the Cox proportional hazard model (Table 3). In the crude analysis, high MLR associated with increased risk of CVD events excluding sudden death in binary analysis as a rank variable when dividing the patients into 2 groups according to median MLR (RR 2.21; 95\% CI, 1.13-4.35) and as a continuous variable per unit of change (RR 6.03; 95\% CI, 1.30-23.66) and change over the whole range (RR 7.10; 95\% CI, 1.32-31.57) When adjusted for age, sex, and diabetes, the risk associated with higher MLR remained significant with RR 2.69 (95\% CI, $1.12-7.14)$ in the binary analysis, RR 6.04 (1.18-26.41) when analyzing changes per unit, and RR 7.12 (1.2035.60) when analyzing changes over the whole range of MLR. Also, high MLR associated with increased risk of any type of CVD events (thus including sudden deaths):

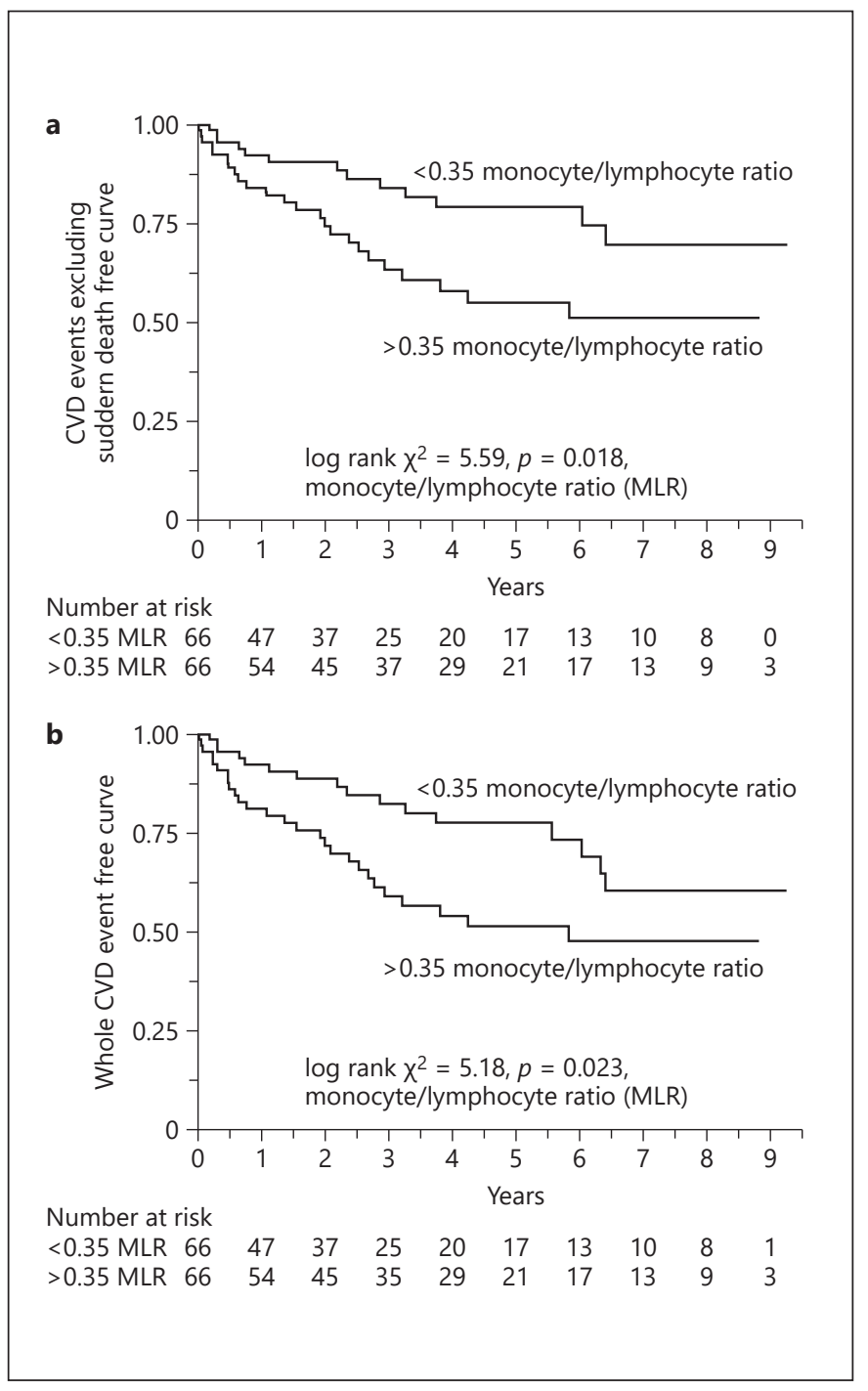

Fig. 1. Kaplan-Meier curves showing the first cardiovascular event from the start of dialysis therapy in 132 incident dialysis patients divided into 2 groups according to median MLR. The median MLR was 0.35 (range 0.27-0.46). a CVD events excluding sudden death. b Total CVD events. MLR, monocyte/lymphocyte ratio; CVD, cardiovascular disease.

in binary analysis as a rank variable (RR 2.02; 95\% CI, 1.09-3.76), and as a continuous variable per unit of change (RR 4.54; 95\% CI, 1.03-17.08), and as change over the whole range (RR 5.22; 95\% CI, 1.03-21.12) in the crude analysis. When adjusted for the same parameters, the risk associated with higher MLR remained significant with RR 2.69 (95\% CI, 1.12-7.14) in the binary analysis, but it was not significant when analyzing MLR changes as a continuous variable. Moreover, because smoking habit and CRP could associate with differential 
Table 3. RRs with $95 \% \mathrm{CI}$ for MLR and other factors predicting CVD events after initiation of dialysis therapy

\begin{tabular}{|c|c|c|c|c|}
\hline \multirow[t]{2}{*}{ Parameter } & \multicolumn{2}{|c|}{ CVD events excluding sudden death } & \multicolumn{2}{|l|}{ All CVD events } \\
\hline & RRs $(95 \% \mathrm{Cl})$ & $p$ value & RRs $(95 \% \mathrm{Cl})$ & $p$ value \\
\hline \multicolumn{5}{|l|}{ Unadjusted } \\
\hline Binary analysis; MLR, >median ${ }^{\dagger}$ & $2.21(1.13-4.35)$ & $0.021 *$ & $2.02(1.09-3.76)$ & $0.026^{*}$ \\
\hline Analysis as continuous variable; MLR (per unit) ${ }^{\dagger+}$ & $6.03(1.30-23.66)$ & $0.015^{*}$ & $4.54(1.03-17.08)$ & $0.034^{*}$ \\
\hline Analysis as continuous variable; MLR (whole) ${ }^{\dagger+\dagger}$ & $7.10(1.32-31.57)$ & $0.015^{*}$ & $5.22(1.03-21.12)$ & $0.015^{*}$ \\
\hline \multicolumn{5}{|l|}{ Model 1} \\
\hline MLR, $>$ median $^{\dagger}$ & $2.44(1.22-4.85)$ & $0.011^{*}$ & $2.21(1.17-4.14)$ & $0.014^{*}$ \\
\hline Age, $>61$ years & $2.21(1.12-4.35)$ & $0.022^{*}$ & $1.82(0.98-3.38)$ & 0.057 \\
\hline Gender, man & $2.55(1.11-5.91)$ & $0.029 *$ & $2.70(1.23-5.90)$ & $0.013^{*}$ \\
\hline Diabetes mellitus, presence & $5.83(2.61-13.0)$ & $<0.0001^{*}$ & $4.58(2.26-9.26)$ & $<0.0001^{*}$ \\
\hline \multicolumn{5}{|l|}{ Model 2} \\
\hline MLR (per unit) ${ }^{\dagger \dagger}$ & $6.04(1.18-26.41)$ & $0.022^{*}$ & $4.35(0.91-17.88)$ & 0.051 \\
\hline MLR (per whole) ${ }^{\dagger+\dagger}$ & $7.12(1.20-35.60)$ & $0.022^{*}$ & $4.98(0.90-35.60)$ & 0.051 \\
\hline Age, $>61$ years & $2.10(1.06-4.15)$ & $0.032 *$ & $1.77(0.96-3.30)$ & 0.069 \\
\hline Gender, man & $2.33(0.99-5.48)$ & 0.052 & $2.51(1.13-5.56)$ & $0.023^{*}$ \\
\hline Diabetes mellitus, presence & $5.62(2.52-12.51)$ & $<0.0001^{*}$ & $4.42(2.19-8.91)$ & $<0.0001^{*}$ \\
\hline \multicolumn{5}{|l|}{ Model 3 (model $2+$ smoking habit + CRP) } \\
\hline$M L R,>$ median $^{\dagger}$ & $2.37(1.19-4.72)$ & $0.014^{*}$ & $2.10(1.12-3.96)$ & $0.022^{*}$ \\
\hline MLR (per unit) $)^{\dagger \dagger}$ & $5.56(1.05-25.71)$ & $0.028^{*}$ & $3.93(0.86-17.99)$ & 0.078 \\
\hline MLR (per whole) ${ }^{\dagger+\dagger}$ & $6.56(1.12-34.57)$ & $0.028^{*}$ & $4.45(0.85-23.41)$ & 0.078 \\
\hline
\end{tabular}

RRs, relative risks; MLR, monocyte/lymphocyte ratio; CVD, cardiovascular disease; $\mathrm{Cl}$, confidence interval. ${ }^{\dagger} \mathrm{RR}$ was calculated by dividing the MLR into 2 groups' ratio as an ordinal variable. ${ }^{\dagger+}$ By MLR as a continuous variable when changing by 1 unit. ${ }^{t+\dagger}$ By MLR as a continuous variable when changing by whole range. ${ }^{*} p<0.05$.

leukocyte count, we checked the RRs of the first CVD event in the Cox proportional hazard model adjusted with those confounders.

The Time until the First Hospitalization due to

Infection after Start of Dialysis Therapy

Kaplan-Meier curves revealed that the period without infections requiring hospitalization was significantly shorter in the high MLR group than in the low MLR group (log-rank test 4.16, $p=0.041$ ) (Fig. 2). Kaplan-Meier curves were converging after 7.5 years but not crossing. Next, we calculated the RRs of infectious hospitalization in the Cox proportional hazard model (Table 4). The crude analysis showed that the patients with high MLR had increased risk of infectious disease hospitalization in binary analysis as a rank variable when dividing the patients into 2 groups according to median MLR (RR 1.91; 95\% CI, 1.01-3.60). This association remained statistically significant also when adjusting for age, sex, and diabetes, with RR 2.44 (95\% CI, 1.22-4.85) in the binary analysis while the analyses of MLR as a continuous variable did not show an increase in the risk of infectious disease hospitalization.

Monocyte/Lymphocyte Ratio in Dialysis Patients

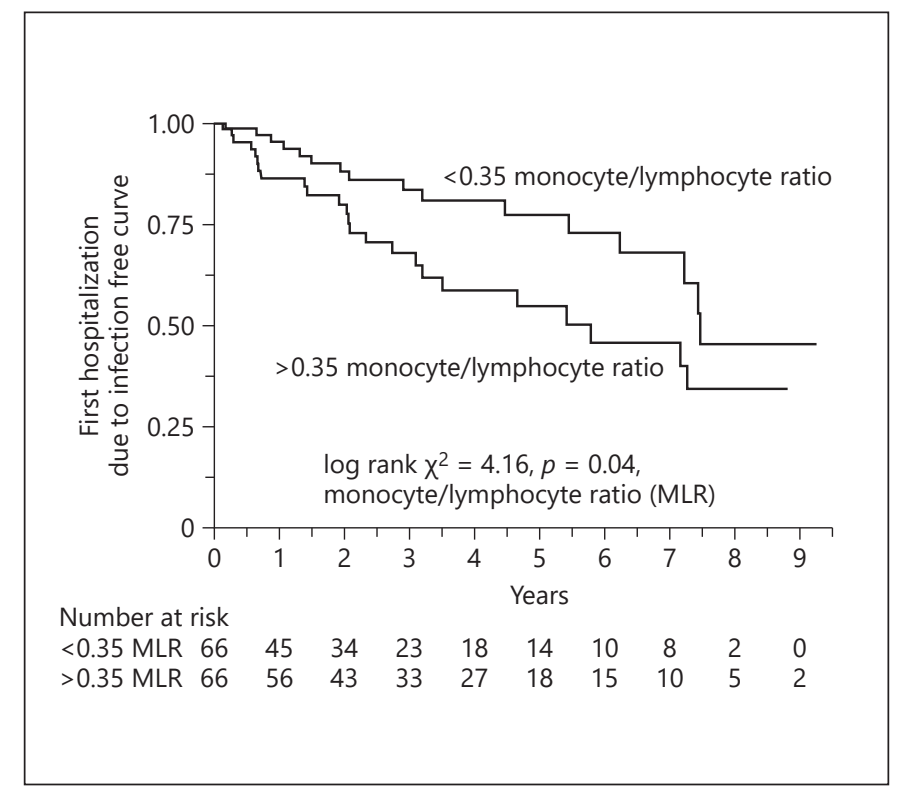

Fig. 2. Kaplan-Meier curves showing the first hospitalization due to infection from the start of dialysis therapy in 132 incident dialysis patients divided into 2 groups according to median MLR. The median MLR was 0.35 (range $0.27-0.46$ ). MLR, monocyte/lymphocyte ratio. 
Table 4. RR and $95 \% \mathrm{Cl}$ for factors predicting the infectious disease hospitalization after initiation of dialysis therapy
Fig. 3. a The cumulative number of cardiovascular events in relation to median MLR. Cardiovascular events increased in patients with high MLR. b Total infection-related hospital stays during the entire observation period in relation to median MLR. Infection-related hospital stays were longer in patients with high MLR. The median MLR was 0.35 (range 0.27-0.4). MLR, monocyte/lymphocyte ratio; CVD, cardiovascular disease.

\begin{tabular}{|c|c|c|}
\hline Parameter & Hazard ratio $(95 \% \mathrm{Cl})$ & $p$ value \\
\hline \multicolumn{3}{|l|}{ Unadjusted } \\
\hline Binary analysis; MLR, $>$ median ${ }^{\dagger}$ & $1.91(1.01-3.60)$ & $0.045^{*}$ \\
\hline Analysis as continuous variable; MLR (per unit) ${ }^{\dagger \dagger}$ & $2.42(0.45-10.77)$ & 0.289 \\
\hline Analysis as continuous variable; MLR (per whole) ${ }^{+\dagger \dagger}$ & $2.62(0.42-13.38)$ & 0.273 \\
\hline \multicolumn{3}{|l|}{ Model 1} \\
\hline MLR, $>$ median $^{\dagger}$ & $2.44(1.22-4.85)$ & $0.026^{*}$ \\
\hline Age, $>61$ years & $2.21(1.12-4.35)$ & 0.187 \\
\hline Gender, man & $2.56(1.10-5.92)$ & $0.014^{*}$ \\
\hline Diabetes mellitus, presence & $5.83(2.61-13.03)$ & $0.003^{*}$ \\
\hline \multicolumn{3}{|l|}{ Model 3 (model $2+$ smoking habit + CRP) } \\
\hline $\mathrm{MLR}_{,}>$median $^{\dagger}$ & $1.99(1.01-3.92)$ & $0.046^{*}$ \\
\hline
\end{tabular}

$\mathrm{RR}$, relative risk; $\mathrm{MLR}$, monocyte/lymphocyte ratio; $\mathrm{Cl}$, confidence interval. ${ }^{\dagger} \mathrm{RR}$ was calculated by dividing the MLR into 2 groups' ratio as an ordinal variable, ${ }^{+\dagger}$ By MLR as a continuous variable when changing by 1 unit. $^{++\dagger}$ By MLR as a continuous variable when changing by whole range. ${ }^{*} p<0.05$.

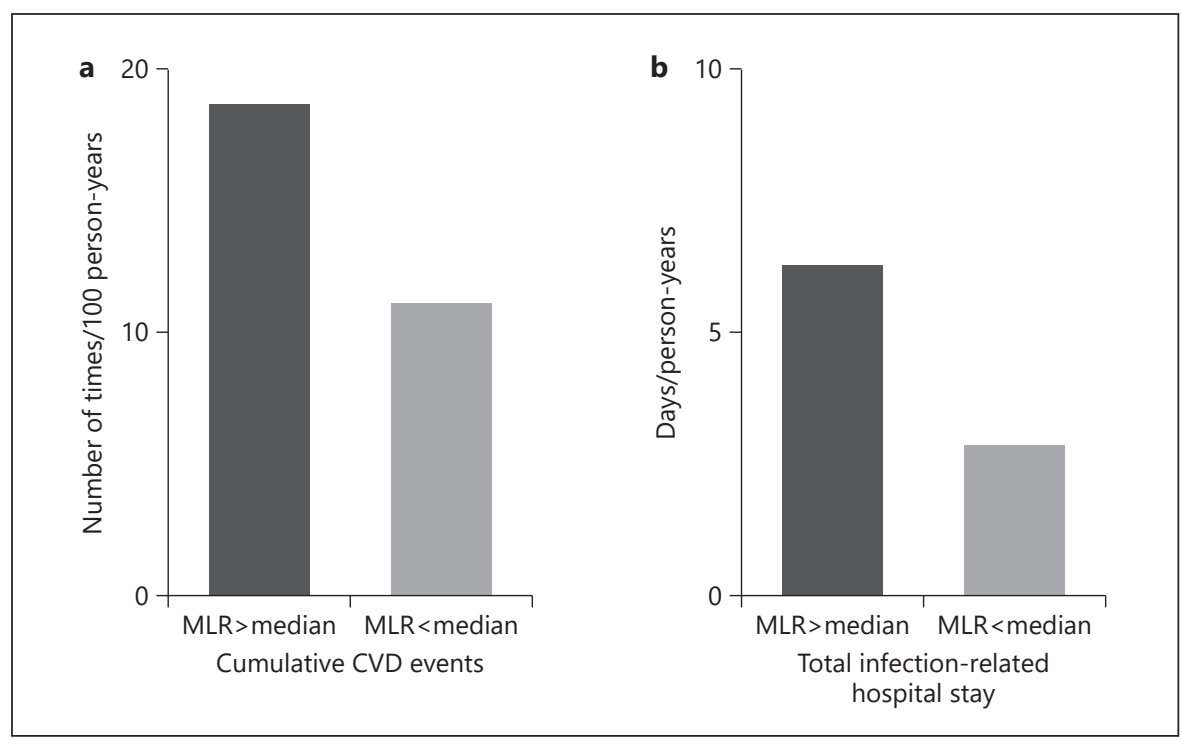

The Cumulative CVD Events and the Duration of Infectious Disease Hospitalization during the Total Observation Period

Forty-three patients experienced CVD events during the observation period: a single event occurred in $24 \mathrm{pa}-$ tients, 9 patients experienced 2 events, 6 patients had 3 events, 4-6 events occurred in each 1 patient, and 1 patient had repeated 9 events. Therefore, a total number of $84 \mathrm{CVD}$ events were recorded during the observation period. The number of CVD events per year was higher in patients with high MLR (18.6 events per 100 personyears) than in patients with low MLR (11.1 events per 100 person-years) (Fig. 3). The duration of infectious hospi- talization per year was longer in patients with high MLR (6.3 days per person-years) than in patients with low MLR (2.8 days per person-years) (Fig. 3 ).

\section{Discussion}

In this study on the prognostic value of MLR as a biomarker for cardiovascular and infectious complications in Japanese incident dialysis patients, the main finding is that a higher baseline MLR was associated with an increased risk of future CVD events and infectious disease hospitalizations. The CVD event-free period and the pe- 
riod without hospitalization for infections were significantly shorter in the high MLR group than in the low MLR group. The RRs of first CVD event and first infectious hospitalization, respectively, were increased in the high MLR group compared to the low MLR group. Furthermore, the cumulative number of CVD events was higher, and the duration of infectious hospitalizations was longer in patients with high versus low baseline MLR.

In dialysis patients, one of the main reasons why CVD remains the leading cause of mortality is premature accelerated atherosclerosis, which involves persistent lowgrade inflammation [19]. Atherosclerosis plaques are characterized by lipid-rich chronic inflammation of the arterial wall, and inflammatory macrophages originating from circulatory monocytes are the most abundant immune cells within plaques [20]. This may explain why an increased blood monocyte count was reported to associate with poor clinical outcomes in patients with chronic kidney disease (CKD). In a study from Japan of 333 prevalent hemodialysis patients, a high monocyte count was associated with CVD-related mortality (accounting for $58.2 \%$ of all deaths) and total long-term mortality [21]. And a large cohort study from the USA found a significant association between higher monocyte count and risk of incident CKD and progression to end-stage renal disease [22]. On the other hand, it is well known that a low lymphocyte count is associated with CVD [23] and overall mortality [24]. Thus, it seems plausible to assume that MLR, that is, the ratio of monocyte count to lymphocyte count, could be more a sensitive predictor than the absolute number of monocyte or lymphocyte counts used separately. Indeed, high MLR has been reported to predict presence of coronary artery disease and its severity in patients undergoing coronary angiography [25], CVD mortality in peritoneal dialysis patients in a retrospective cohort study [15], and overall and CVD mortality in prevalent maintenance hemodialysis patients [14]. To the best of our knowledge, the present study is the first to demonstrate that high MLR is associated with increased risk of CVD events in incident dialysis patients in a long-term prospective cohort.

In a previous study, we found that a higher NLR indicated an increased risk of CVD in incident dialysis patients [13]. Likewise, NLR was found to be a biomarker for CVD in studies of large sample size [26] as confirmed also by meta-analysis [27] of studies in CKD populations. However, no such association was observed when analyzing the dataset of the present study (data not shown). We also did not find an association between high NLR and

Monocyte/Lymphocyte Ratio in Dialysis Patients infection events in the previous dataset or in the present study (data not shown). As to possible reasons for the lack of a relationship between NLR and CVD events in the current study, and instead appearance of a relationship between MLR and CVD events, this difference may be related to the larger sample size ( $n=132$ vs. 86 patients) and longer observation period (median 48.7 months vs. 38.7 months) in the current study than our previous study [13]. In addition, the fundamentally functional differences between neutrophils and monocytes might also contribute. Although both cell types originate from hematopoietic stem cells and progenitor cells in the bone marrow, the main function of neutrophils is as a first-line defense cell under acute inflammation while macrophages have complex roles in homeostasis, tissue repair, and immunity, acting in various tissues [28]. Macrophages have important roles in chronic diseases, including atherosclerosis, asthma, inflammatory bowel disease, rheumatoid arthritis, and fibrosis, and their contributions vary in different disease stages, being controlled by many factors [29]. Thus, one may speculate that this discrepancy could be due to the longer follow-up period in the current study as monocytes conceivably are more influenced by a state of chronic inflammation at baseline than neutrophils.

Next, as regards to the relation of MLR with infection events, a recent systematic review of the clinical utility of different peripheral blood leukocyte ratios as biomarkers of diagnosis or prognosis in bacterial and viral infections, malaria, and critical illness due to sepsis showed that while NLR associates both with bacterial infections, including pneumonia, urinary tract infection, diabetic foot infection and sepsis, and with viral infections, MLR was only associated with viral infections, especially influenza [30]. However, both MLR and NLR were reported to be useful diagnostic tools to identify bacterial infections in patients with fever [31]. Thus, one may speculate that high MLR might associate with asymptomatic inflammation while high NLR might associate with inflammation caused by current illness including acute infections. The observation in the present study of an association in dialysis patients between MLR and long-term risk of future infection events - together with an association between MLR and future CVD events - is a novel finding.

Our observations may reflect the plausible relationship in dialysis patients between CVD and infections, 2 conditions that share many risk factors, including a chronic inflammatory state and the uremic milieu, in patients who in general are immunocompromised [12]. A link between CVD and infections could be expected 
due to the immune dysfunction in uremia where the underlying conditions, on the one hand, induce immune suppression resulting in increased susceptibility to infections and, on the other hand, drive immune activation resulting in inflammation and atherosclerosis [11]. This is supported by studies in clinical settings showing that infection and CVD may induce subsequent CVD and infection events, and vice versa. One study showed that the risks of second CVD events increased after infections in patients with a history of CVD in a large database study in the UK [32]. In 2 community-based cohorts in the USA, hospitalization for pneumonia increased the risk of CVD [33]. Infectious hospitalization increased the subsequent risk of cardiovascular events in older dialysis patients [34]. Moreover, as an atherosclerotic plaque lesion is composed of activated macrophages originating from monocytes, innate immune activation is a central process in the pathogenesis of atherosclerosis [35]. In this process, infectious agents, such as bacterial fragments, are strong stimulants via toll-like receptor signaling [36, 37]. Although we did not investigate a cause-and-effect sequence between CVD and infection in this study, MLR could be a candidate for a link between them.

Some limitations of this study should be acknowledged. First, the number of patients and events are limited, and many potential cofounders could not be taken into account. Second, due to the observational design of the study, we cannot conclude on causality. Third, we checked MLR only once - close to the start of dialysis while serial measurements would have been more informative. Finally, it is a limitation that the study was not powered to analyze possible association of MLR with mortality in a meaningful way due to the age limitation at enrollment and the good prognosis of Japanese HD patients with relatively few deaths. Strengths of the study include the long-term follow-up and careful registration of outcome measures in incident dialysis patients with no patient lost to follow-up and the novel finding that an increased MLR is a risk marker that may link cardiovascular and infectious complications in dialysis patients.

\section{Conclusion}

This study demonstrated that a higher MLR was associated with an increased risk of both CVD events and infectious disease hospitalization in incident dialysis patients. Further studies are however needed to confirm these results and clarify discrepancies and inconsistencies with previous results, and to explore whether the observed associations may reflect potential causal pathways. Nevertheless, our findings may suggest that MLR might be a useful biomarker - readily available in routine clinical practice - for predicting the risk of future CVD events and infections in dialysis patients.

\section{Acknowledgments}

We would like to thank Yoshinari Tsuruta and Kana Nakauchi from Meiyo Clinic, and Kunio Morozumi, and Kaoru Yasuda from Masuko Memorial Hospital for enrolling patients. And we would like to thank Editage (www.editage.jp) for English language editing.

\section{Statement of Ethics}

The study was approved by the Ethics Committee of Nagoya University Graduate School of Medicine (No. 2012-0241). Informed consent for participation was obtained from all patients.

\section{Conflict of Interest Statement}

B.L. is employed by Baxter Healthcare Corporation. Baxter Novum is the result of a grant from Baxter Healthcare Corporation to Karolinska Institutet. Other authors declare no conflicts of interest.

\section{Funding Sources}

This study was supported in part by a Grant-in-Aid for Progressive Renal Diseases Research, Research on Rare and Intractable Disease, and from the Ministry of Health, Labor and Welfare of Japan.

\section{Author Contributions}

R.M. was primarily responsible for researching and writing the article. S.K. was responsible for the research idea, the study concept, design and organization of the original cohort, analysis, interpretation of data, and writing this article. B.L. T.I. and T.K. took part in composing the cohort with advising the research idea and the study concept, as well as writing this article. A.R.Q. took part in the statistical analysis. S.M. was also responsible for the research idea, the study concept, design, and organization of the original cohort as a principal investigator as well as supervising the writing of the article.

\section{Data Availability Statement}

All data generated or analyzed during this study are included in this article. Further inquiries can be directed to the corresponding author. 


\section{References}

1 Foley RN, Collins AJ. The USRDS: what you need to know about what it can and can't tell us about ESRD. Clin J Am Soc Nephrol. 2013 May;8(5):845-51.

2 United States Renal Data System. 2019 USRDS annual data report: epidemiology of kidney disease in the United States. Bethesda, MD: National Institutes of Health, National Institute of Diabetes and Digestive and Kidney Diseases; 2019.

3 Foley RN, Parfrey PS, Sarnak MJ. Epidemiology of cardiovascular disease in chronic renal disease. J Am Soc Nephrol. 1998 Dec;9(12 Suppl):S16-23.

4 Stack AG, Neylon AM, Abdalla AA, Hegarty A, Hannigan A, Cronin CJ, et al. Declining mortality rates despite increases in clinical coronary artery disease among US dialysis patients: a national registry study. Am J Nephrol. 2013;38(1):66-74.

5 Inampudi C, Akintoye E, Bengaluru Jayanna M, Asleh R, Briasouli A, Alvarez P, et al. Trends in in-hospital mortality, length of stay, nonroutine discharge, and cost among endstage renal disease patients on dialysis hospitalized with heart failure (2001-2014). J Card Fail. 2019 Jul;25(7):524-33.

6 Hanafusa N, Nakai S, Iseki K, Tsubakihara Y. Japanese society for dialysis therapy renal data registry-a window through which we can view the details of Japanese dialysis population. Kidney Int Suppl. 2015 Jun;5(1):15-22.

7 de Jager DJ, Grootendorst DC, Jager KJ, van Dijk PC, Tomas LM, Ansell D, et al. Cardiovascular and noncardiovascular mortality among patients starting dialysis. JAMA. 2009 Oct 28;302(16):1782-9.

8 Collins AJ, Foley RN, Gilbertson DT, Chen SC. United States Renal Data System public health surveillance of chronic kidney disease and end-stage renal disease. Kidney Int Suppl. 2015 Jun;5(1):2-7.

9 Wakasugi M, Kawamura K, Yamamoto S, Kazama JJ, Narita I. High mortality rate of infectious diseases in dialysis patients: a comparison with the general population in Japan. Ther Apher Dial. 2012 Jun;16(3):226-31.

10 Jager KJ, Lindholm B, Goldsmith D, Fliser D, Wiecek A, Suleymanlar G, et al. Cardiovascular and non-cardiovascular mortality in dialysis patients: where is the link? Kidney Int Suppl. 2011 Jun;1(1):21-3.

11 Kato S, Chmielewski M, Honda H, PecoitsFilho R, Matsuo S, Yuzawa Y, et al. Aspects of immune dysfunction in end-stage renal disease. Clin J Am Soc Nephrol. 2008 Sep;3(5): 1526-33.

12 de Jager DJ, Vervloet MG, Dekker FW. Noncardiovascular mortality in CKD: an epidemiological perspective. Nat Rev Nephrol. 2014 Apr;10(4):208-14.
13 Abe T, Kato S, Tsuruta Y, Sugiura S, Katsuno T, Kosugi T, et al. Neutrophil/lymphocyte ratio as a predictor of cardiovascular events in incident dialysis patients: a Japanese prospective cohort study. Clin Exp Nephrol. 2015 Aug;19(4):718-24.

14 Xiang F, Chen R, Cao X, Shen B, Liu Z, Tan $\mathrm{X}$, et al. Monocyte/lymphocyte ratio as a better predictor of cardiovascular and all-cause mortality in hemodialysis patients: a prospective cohort study. Hemodial Int. 2018 Jan; 22(1):82-92.

15 Wen Y, Zhan X, Wang N, Peng F, Feng X, Wu $\mathrm{X}$. Monocyte/lymphocyte ratio and cardiovascular disease mortality in peritoneal dialysis patients. Mediators Inflamm. 2020;2020: 9852507.

16 Kato S, Lindholm B, Yuzawa Y, Tsuruta Y, Nakauchi K, Yasuda K, et al. High ferritin level and malnutrition predict high risk of infection-related hospitalization in incident dialysis patients: a Japanese Prospective Cohort Study. Blood Purif. 2016;42(1):56-63.

17 Kato S, Shiels PG, McGuinness D, Lindholm B, Stenvinkel P, Nordfors L, et al. Telomere attrition and elongation after chronic dialysis initiation in patients with end-stage renal disease. Blood Purif. 2016;41(1-3):25-33.

18 Kato S, Lindholm B, Qureshi AR, Mukai H, Yuzawa Y, Maruyama S. Inter-leg systolic blood pressure difference predicts cardiovascular events and mortality in incident hemodialysis patients. Int J Artif Organs. 2020 Apr; 43(4):217-24.

19 Dai L, Golembiewska E, Lindholm B, Stenvinkel P. End-stage renal disease, inflammation and cardiovascular outcomes. Contrib Nephrol. 2017;191:32-43.

20 Groh L, Keating ST, Joosten LAB, Netea MG, Riksen NP. Monocyte and macrophage immunometabolism in atherosclerosis. Semin Immunopathol. $2018 \mathrm{Feb}$;0(2):203-14.

21 Kato A, Takita T, Furuhashi M, Maruyama Y, Kumagai H, Hishida A. Blood monocyte count is a predictor of total and cardiovascular mortality in hemodialysis patients. Nephron Clin Pract. 2008;110(4):c235-43.

22 Bowe B, Xie Y, Xian H, Li T, Al-Aly Z. Association between monocyte count and risk of incident CKD and progression to ESRD. Clin J Am Soc Nephrol. 2017 Apr 3;12(4):603-13.

23 Nunez J, Minana G, Bodi V, Nunez E, Sanchis J, Husser O, et al. Low lymphocyte count and cardiovascular diseases. Curr Med Chem. 2011;18(21):3226-33.

24 Zidar DA, Al-Kindi SG, Liu Y, Krieger NI, Perzynski AT, Osnard M, et al. Association of lymphopenia with risk of mortality among adults in the US general population. JAMA Netw Open. 2019 Dec 2;2(12):e1916526.

25 Ji H, Li Y, Fan Z, Zuo B, Jian X, Li L, et al. Monocyte/lymphocyte ratio predicts the severity of coronary artery disease: a syntax score assessment. BMC Cardiovasc Disord. 2017 Mar 31;17(1):90.
26 Yuan Q, Wang J, Peng Z, Zhou Q, Xiao X, Xie $\mathrm{Y}$, et al. Neutrophil-to-lymphocyte ratio and incident end-stage renal disease in Chinese patients with chronic kidney disease: results from the Chinese Cohort Study of chronic kidney disease (C-STRIDE). J Transl Med. 2019 Mar 15;17(1):86.

27 Chen D, Xiao D, Guo J, Chahan B, Wang Z. Neutrophil-lymphocyte count ratio as a diagnostic marker for acute kidney injury: a systematic review and meta-analysis. Clin Exp Nephrol. 2020 Feb;24(2):126-35.

28 Yvan-Charvet L, Bonacina F, Guinamard RR, Norata GD. Immunometabolic function of cholesterol in cardiovascular disease and beyond. Cardiovasc Res. 2019 Jul 1;115(9): 1393-407.

29 Wynn TA, Chawla A, Pollard JW. Macrophage biology in development, homeostasis and disease. Nature. 2013 Apr 25;496(7446): 445-55.

30 Russell CD, Parajuli A, Gale HJ, Bulteel NS, Schuetz P, de Jager CPC, et al. The utility of peripheral blood leucocyte ratios as biomarkers in infectious diseases: a systematic review and meta-analysis. J Infect. 2019 May;78(5): 339-48.

31 Naess A, Nilssen SS, Mo R, Eide GE, Sjursen $\mathrm{H}$. Role of neutrophil to lymphocyte and monocyte to lymphocyte ratios in the diagnosis of bacterial infection in patients with fever. Infection. 2017 Jun;45(3):299-307.

32 Smeeth L, Thomas SL, Hall AJ, Hubbard R, Farrington P, Vallance P. Risk of myocardial infarction and stroke after acute infection or vaccination. N Engl J Med. 2004 Dec 16; 351(25):2611-8.

33 Corrales-Medina VF, Alvarez KN, Weissfeld LA, Angus DC, Chirinos JA, Chang CC, et al. Association between hospitalization for pneumonia and subsequent risk of cardiovascular disease. JAMA. 2015 Jan 20;313(3):26474.

34 Dalrymple LS, Mohammed SM, Mu Y, Johansen KL, Chertow GM, Grimes B, et al. Risk of cardiovascular events after infection-related hospitalizations in older patients on dialysis. Clin J Am Soc Nephrol. 2011 Jul;6(7):170813.

35 Heine GH, Ortiz A, Massy ZA, Lindholm B, Wiecek A, Martínez-Castelao A, et al. Monocyte subpopulations and cardiovascular risk in chronic kidney disease. Nat Rev Nephrol. 2012 Mar 13;8(6):362-9.

36 Ghattas A, Griffiths HR, Devitt A, Lip GY, Shantsila E. Monocytes in coronary artery disease and atherosclerosis: where are we now? J Am Coll Cardiol. 2013 Oct 22;62(17): 1541-51.

37 Moore KJ, Sheedy FJ, Fisher EA. Macrophages in atherosclerosis: a dynamic balance. Nat Rev Immunol. 2013 Oct;13(10):709-21. 